\title{
Universal microscopic correlation functions for products of independent Ginibre matrices
}

\author{
Gernot AKemann $^{1}$ and Zdzislaw Burda ${ }^{2}$ \\ ${ }^{1}$ Department of Physics, Bielefeld University, \\ Postfach 100131, D-33501 Bielefeld, Germany \\ ${ }^{2}$ Marian Smoluchowski Institute of Physics, Jagellonian University, \\ Reymonta 4, 30-059 Krákow, Poland
}

\begin{abstract}
We consider the product of $n$ complex non-Hermitian, independent random matrices, each of size $N \times N$ with independent identically distributed Gaussian entries (Ginibre matrices). The joint probability distribution of the complex eigenvalues of the product matrix is found to be given by a determinantal point process as in the case of a single Ginibre matrix, but with a more complicated weight given by a Meijer $G$-function depending on $n$. Using the method of orthogonal polynomials we compute all eigenvalue density correlation functions exactly for finite $N$ and fixed $n$. They are given by the determinant of the corresponding kernel which we construct explicitly. In the large- $N$ limit at fixed $n$ we first determine the microscopic correlation functions in the bulk and at the edge of the spectrum. After unfolding they are identical to that of the Ginibre ensemble with $n=1$ and thus universal. In contrast the microscopic correlations we find at the origin differ for each $n>1$ and generalise the known Bessel-law in the complex plane for $n=2$ to a new hypergeometric kernel ${ }_{0} F_{n-1}$.
\end{abstract}




\section{Introduction}

The topic of random matrix theory (RMT) enjoys an increasing number of applications in physics, mathematics and other sciences, and we refer to [1] for a recent compilation. This statement holds both for problems with real eigenvalues as well as with complex eigenvalues. In the latter case the classical ensemble was introduced by Ginibre [2] who considered complex non-Hermitian matrices $X$ of size $N \times N$, with all matrix elements having independent normal distributions. However, for certain applications it is not sufficient to introduce a single random matrix, e.g. when considering transfer matrices. The problem of studying such products of random matrices is as old as RMT itself and was introduced by Furstenberg and Kesten [3]. Applications for products of random matrices range from combinatorics [4] over Lyapunov exponents in diffusion problems [5] to Quantum Chromodynamics [6] to name a few recent works.

One problem one has to face is that the product matrix often has less symmetry than the individual matrices. For example the product $P$ of two Hermitian matrices is in general no longer Hermitian, and thus acquires a complex spectrum. Therefore in the literature two types of products have been considered, those which are Hermitised by considering $P^{\dagger} P$ with real spectra, and those which are studying the complex eigenvalues of $P$ itself. In this paper we will consider the latter problem in the simplest setting, by multiplying a fixed number $n$ of independent Ginibre matrices: $P_{n}=X_{1} X_{2} \ldots X_{n}$. This problem has found a renewed interest in recent years in the mathematics and physics literature. In particular the mean or macroscopic density of complex eigenvalues given by the $n$-th power of the circular law was derived independently using diagrammatic methods [7, singular values [8] or empirical spectral distributions [7, 9]. Moreover it was shown that the product of a wide class of non-Hermitian Wigner matrices with independent identically distributed (iid) non-Gaussian entries has the same spectral distribution in the large- $N$ limit as the product of Gaussian matrices [10]. The same macroscopic universality was observed numerically in [9] multiplying matrices from different symmetry classes.

The aim of the present paper is to provide more detailed information for the product matrix, by exactly solving the problem at finite matrix size $N$ first, using orthogonal polynomials in the complex plane. This goal was previously achieved for $n=2$ complex non-Hermitian [6], quaternion real [1] and real asymmetric matrices [12]. These can be considered as non-Hermitian generalisations of WishartLaguerre ensembles, see [13] for a review. From [6] we will borrow the idea of parameterising the $n$ matrices $X_{j}$ in order to compute the joint probability distribution function (jpdf) of $P_{n}$. Our approach enables us to identify various large- $N$ limits and to resolve the fluctuations in 3 different regions: the origin, bulk and edge. A new origin limit was previously found in [6] for $n=2$, see also [14] for gap probabilities in that ensemble. The limiting microscopic density at the edge for $n=2$ was shown in [15] to coincide with that of the Ginibre ensemble $n=1$ [16, 17], and was conjectured to universally hold for arbitrary $n$, based on numerics and including rectangular matrices with various symmetries as well [9]. The same universal edge scaling limit can also be found for truncations of unitary matrices [18], sub-unitary matrices [19], and for the induced Ginibre ensemble [20].

We find that at the origin each $n>1$ leads to a new class of hypergeometric kernels, whereas in the bulk and at the edge the correlations agree for all $n$ with the Ginibre ensemble and are thus universal. Our findings add another facet to the property of universality of correlation functions in the complex plane. This question was answered for a single matrix, either with non-Gaussian invariant distributions in the bulk [21], or with non-invariant iid elements 22 in the bulk and at the edge.

The paper is organised as follows. In the next section 2 we briefly list the main results obtained in this paper. A detailed derivation of the jpdf is given in section 3 , including our matrix parametrisation and the computation of the weight function in the complex plane. Section 4 provides the complete solution for finite- $N$ at fixed $n$ for all eigenvalue density correlation functions in terms of orthogonal 
polynomials and their kernel. The following sections are dealing with the large- $N$ limit, first for the density at large matrix size and large argument in section 5. Section 6 is devoted to the three microscopic limits mentioned earlier, before concluding in section 7 . Several technical details are reported in the appendices $\mathrm{A}-\mathrm{D}$.

\section{Main results}

Given the product $P_{n}$ of $n$ independent matrices $X_{j}, j=1, \ldots, n$, each of size $N \times N$ drawn from the Ginibre ensemble with Gaussian distribution proportional to $\exp \left[-\operatorname{Tr} X_{j}^{\dagger} X_{j}\right]$,

$$
P_{n} \equiv X_{1} X_{2} \ldots X_{n}
$$

The partition function $Z_{n}$ can be expressed as an integral of the joint probability distribution function $\mathcal{P}_{j p d f}$ of the complex eigenvalues $z_{j}, j=1, \ldots, N$ of $P_{n}$, given by

$$
Z_{n}=C_{n} \int \prod_{a=1}^{N}\left(d^{2} z_{a} w_{n}\left(z_{a}\right)\right) \prod_{b>a}^{N}\left|z_{b}-z_{a}\right|^{2} \equiv \int \prod_{a=1}^{N} d^{2} z_{a} \mathcal{P}_{j p d f}(\{z\}),
$$

where $C_{n}$ is some known constant. The weight function $w_{n}(z)$ that depends only on the modulus is given by the so-called Meijer $G$-function

$$
w_{n}(z)=\pi^{n-1} G_{0 n}^{n 0}\left(\left.\overline{\overrightarrow{0}}|| z\right|^{2}\right) .
$$

The corresponding kernel of polynomials orthonormal with respect to that weight reads

$$
K_{N}^{(n)}\left(z_{i}, z_{j}\right)=\sqrt{w_{n}\left(z_{i}\right) w_{n}\left(z_{j}\right)} \sum_{k=0}^{N-1} \frac{1}{(\pi k !)^{n}}\left(z_{i} z_{j}^{*}\right)^{k} .
$$

The $k$-point density correlation functions then easily follow to be the determinant of that kernel,

$$
\begin{aligned}
R_{k}^{(n)}\left(z_{1}, \ldots, z_{k}\right) & \equiv \frac{N !}{(N-k) !} \frac{1}{Z_{n}} \int d^{2} z_{k+1} \ldots d^{2} z_{N} \mathcal{P}_{j p d f}(\{z\}) \\
& =\operatorname{det}_{1 \leq i, j \leq k}\left[K_{N}^{(n)}\left(z_{i}, z_{j}\right)\right] .
\end{aligned}
$$

After briefly summarising our results for both finite $n$ and $N$ which are new for $n>2$ we turn to the large- $N$ limits.

For large $N$ and large arguments $|z| \gg 1$ the eigenvalue density behaves as

$$
R_{1}^{(n)}(z)=K_{N}^{(n)}(z, z) \approx \frac{|z|^{\frac{2}{n}-2}}{n \pi} \frac{1}{2} \operatorname{erfc}\left(\frac{\sqrt{n}\left(|z|^{2 / n}-N\right)}{\sqrt{2}|z|^{1 / n}}\right) .
$$

From this the mean or macroscopic large- $N$ density can be read off:

$$
\rho_{\text {macro }}^{(n)}(w) \equiv \lim _{N \rightarrow \infty} \frac{1}{N} N^{n} R_{1}^{(n)}\left(z=N^{n / 2} w\right)=\frac{|w|^{\frac{2}{n}-2}}{n \pi} \Theta(1-|w|) .
$$

This result was previously derived in [7, 8, 10] and we have rescaled variables such that the support is now the unit disc. Our new results are for the microscopic limit at the origin, in the bulk and at 
the edge of the spectrum. We first quote the latter, by zooming into the region around the edge of the support, which is $z \approx N^{\frac{n}{2}}$ in eq. (2.7):

$$
\rho_{\text {edge }}^{(n)}(\xi) \equiv \lim _{N \rightarrow \infty} n N^{n-1} R_{1}^{(n)}\left(z=N^{n / 2}(1+\xi \sqrt{n / N}) \mathrm{e}^{i \phi}\right)=\frac{1}{2 \pi} \operatorname{erfc}(\sqrt{2} \xi)
$$

This result depending only on the radial distance from the edge is universal in the sense that it agrees for all $n$ with that of the Ginibre ensemble [16, 17] with $n=1$ (see also [23]), and the non-Hermitian Wishart ensemble [15] with $n=2$ derived previously.

For the bulk scaling limit we first have to unfold, in order to have a flat density locally. In our case this can be obtained by a simple rescaling $\xi=\sqrt{n} z^{1 / n}$,

$$
\widehat{R}_{1}^{(n)}(\xi)=\frac{1}{n} z^{2-\frac{2}{n}} R_{1}^{(n)}\left(z=(\xi / \sqrt{n})^{n}\right)
$$

and correspondingly for higher correlation functions. Taking the scaling limit $1 \ll \xi \lesssim \sqrt{N}$ to be in the bulk we obtain the following answer for the asymptotic kernel

$$
\begin{aligned}
K_{\text {bulk }}^{(n)}\left(\xi_{i}, \xi_{j}\right) & \equiv \lim _{N \rightarrow \infty ;\left|\xi_{i, j}\right| \gg 1} n^{2-n}\left|\xi_{i} \xi_{j}\right|^{n-1} K_{N}^{(n)}\left(z_{i}=\left(\xi_{i} / \sqrt{n}\right)^{n}, z_{j}=\left(\xi_{j} / \sqrt{n}\right)^{n}\right) \\
& =\frac{1}{\pi}\left(\frac{\xi_{i} \xi_{j}^{*}}{\left|\xi_{i} \xi_{j}\right|}\right)^{(1-n) / 2} \exp \left[-\frac{1}{2}\left(\left|\xi_{i}\right|^{2}+\left|\xi_{j}\right|^{2}+\xi_{i} \xi_{j}^{*}\right)\right] .
\end{aligned}
$$

This is equal to the rescaled universal kernel in the bulk of the Ginibre ensemble $n=1$, in which the bulk and origin limits coincide. The phase factor in front of the exponential is irrelevant as it drops out after taking the determinant in eq. (2.6).

Finally let us emphasise that there exists a third microscopic large- $N$ limit at the origin which differs for every $n>1$. Its kernel is simply given by extending the sum in eq. (2.4) to infinity, leading to a hypergeometric function:

$$
K_{\text {origin }}^{(n)}\left(z_{i}, z_{j}\right) \equiv \lim _{N \rightarrow \infty} K_{N}^{(n)}\left(z_{i}, z_{j}\right)=\sqrt{w_{n}\left(z_{i}\right) w_{n}\left(z_{j}\right)}{ }_{0} F_{n-1}\left(-; \overrightarrow{1} ; z_{i} z_{j}^{*}\right) \pi^{-n}, n>1 .
$$

Here we have $\left|z_{i}\right|=\mathcal{O}(1)$. For the Ginibre ensemble $n=1$ the limiting kernel is given by that of the bulk limit above, whereas for $n=2$ one has a $K$-Bessel function for the weight times an $I$-Bessel function from the sum in the kernel [6], which was also investigated in [14] including its Fredholm determinant.

\section{Derivation of the joint eigenvalue distribution}

\subsection{Outline of the method}

We are interested in statistical properties of eigenvalues of the product

$$
P_{n}=X_{1} X_{2} \ldots X_{n}
$$

of $n$ independent Ginibre matrices of dimensions $N \times N$. The partition function of independent matrices factorises into a product of independent partition functions for each matrix. We however write it in a compact way

$$
Z_{n}=\prod_{j=1}^{n} \int\left|D X_{j}\right| \mathrm{e}^{-\operatorname{Tr} X_{j}^{\dagger} X_{j}}=\int|D X| \mathrm{e}^{-\operatorname{Tr} \sum_{j=1}^{n} X_{j}^{\dagger} X_{j}}
$$


because we are interested in the eigenvalues of $P_{n}$. In this notation $D X$ is the Euclidean volume form (external product of all independent one-forms) and $|D X|$ is the corresponding unoriented volume element:

$$
\begin{aligned}
|D X|=\prod_{j=1}^{n}\left|D X_{j}\right| & =\prod_{j=1}^{n} \prod_{a, b=1}^{N}\left(\left(d \operatorname{Re} X_{j}\right)_{a b}\left(d \operatorname{Im} X_{j}\right)_{a b} / 2 \pi\right) \\
& =\prod_{j=1}^{n} \prod_{a, b=1}^{N}\left(\left(d X_{j}\right)_{a b}\left(d X_{j}\right)_{a b}^{*} / 4 \pi\right) .
\end{aligned}
$$

The star denotes the complex conjugate. The index $j$ runs over the set of matrices $j=1,2, \ldots, n$ and $a$ and $b$ over rows and columns of the matrix $a=1, \ldots, N$ and $b=1, \ldots, N$.

Our first goal is to rewrite the integrand of (3.2) in new variables that can be divided into two classes: the first one comprising variables that are directly related to the eigenvalues of $P_{n}$ and the second one comprising variables independent of the eigenvalues of $P_{n}$. Having done that we can try to integrate out the variables of the second class to obtain an integrand depending only on eigenvalues of $P_{n}$. We obtain in this way the joint probability distribution function $\mathcal{P}_{j p d f}$ for eigenvalues of $P_{n}$. We basically follow the Dyson procedure but introduce some pertinent modifications that enable us to handle the case of eigenvalues of the product matrix $P_{n}$ in eq. (3.1).

It is convenient to consider a cyclic block matrix $B$, following [7]:

$$
B=\left(\begin{array}{cccccc}
0 & X_{1} & 0 & 0 & \ldots & 0 \\
0 & 0 & X_{2} & 0 & \ldots & 0 \\
0 & 0 & 0 & X_{3} & \ldots & 0 \\
& & & & \ldots & \\
0 & 0 & 0 & 0 & \ldots & X_{n-1} \\
X_{n} & 0 & 0 & 0 & \ldots & 0
\end{array}\right) .
$$

Each block $X_{j}$ in $B$ is of dimensions $N \times N$, so the whole matrix has dimensions $n N \times n N$. One can easily see that the $n$-th power $B^{n}$ has identical eigenvalues as $P_{n}$, but each being $n$ times degenerate. Indeed, $B^{n}$ is a block diagonal matrix. The first diagonal block is $X_{1} X_{2} \ldots X_{n}$, the second one is $X_{2} X_{3} \ldots X_{n} X_{1}$, and all others are products of consecutive cyclic permutations of $X_{1}, X_{2}, \ldots, X_{n}$. Each diagonal block of $B^{n}$ has the same eigenvalues as $P_{n}$, so therefore each eigenvalue of $P_{n}$ appears in $B^{n}$ in $n$ copies.

\subsection{Change of variables}

In this section we change variables, as outlined above, and find the corresponding Jacobian. Without loss of generality we illustrate our method for $n=3$. We use the following parametrisation of the block matrix:

$$
\begin{aligned}
B & =\left(\begin{array}{ccc}
0 & X_{1} & 0 \\
0 & 0 & X_{2} \\
X_{3} & 0 & 0
\end{array}\right) \\
& =\left(\begin{array}{ccc}
U_{1} & 0 & 0 \\
0 & U_{2} & 0 \\
0 & 0 & U_{3}
\end{array}\right)\left(\begin{array}{ccc}
0 & \Lambda_{1}+T_{1} & 0 \\
0 & 0 & \Lambda_{2}+T_{2} \\
\Lambda_{3}+T_{3} & 0 & 0
\end{array}\right)\left(\begin{array}{ccc}
U_{1}^{-1} & 0 & 0 \\
0 & U_{2}^{-1} & 0 \\
0 & 0 & U_{3}^{-1}
\end{array}\right) .
\end{aligned}
$$

This is a generalisation of Osborn's idea who considered the chiral block matrix for $n=2[6]$. It is also known as $Q Z$ or generalised Schur decomposition, see e.g. 24] section 7.7. The diagonal block unitary 
matrix $U$ on the left hand side has three diagonal unitary blocks $U_{j}$ of dimensions $N \times N$ restricted to the right coset $U(N) / U(1)^{N}$. Each block $\Lambda_{j}$ is a $N \times N$ diagonal matrix $\Lambda_{j}=\operatorname{diag}\left(x_{j 1}, \ldots, x_{j N}\right)$. Each block $T_{j}$ is an $N \times N$ strictly upper triangular matrix. One should note that the eigenvalues of $B^{3}$ (and thus also of $P_{3}$ ) are equal to $x_{1 a} x_{2 a} x_{3 a}$ for $a=1, \ldots, N$.

In the original representation, the block matrix $B$ is parametrised by the elements of the matrices $X_{j}$. Each matrix $X_{j}$ has $N^{2}$ elements, and each element is a complex variable (two real degrees of freedom). So altogether there are $6 N^{2}$ real degrees of freedom. This number matches the number of degrees of freedom of the parametrisation on the right-hand-side: each $U_{j}$ has $N(N-1)$ real parameters, each $\Lambda_{j}$ has $2 N$ and each $T_{j}$ has $N(N-1)$. It makes $2 N^{2}$ times 3 , that is $6 N^{2}$ real degrees of freedom as in the original parametrisation. In Appendix A we calculate the Jacobian for the change of variables (3.5) and integrate out the $T_{j}$ 's and $U_{j}$ 's. The corresponding integrals factorise and the constants can be read off from the known Ginibre ensemble. We are left with an integral

$$
Z_{3}=C_{3} \int \prod_{j=1}^{n} \prod_{a=1}^{N} d^{2} x_{j a} \prod_{b>a}^{N}\left|x_{1 b} x_{2 b} x_{3 b}-x_{1 a} x_{2 a} x_{3 a}\right|^{2} \mathrm{e}^{-\sum_{j=1}^{n} \sum_{a=1}^{N}\left|x_{j a}\right|^{2}}, \quad C_{3}=\frac{1}{\left(N ! G[N+1] \pi^{N}\right)^{3}},
$$

that depends on eigenvalues of the three matrices in the product. Here $G[N+1]=\prod_{k=0}^{N-1} k$ ! is the Barnes $G$-function. As discussed the $z_{a}=x_{1 a} x_{2 a} x_{3 a}, a=1, \ldots, N$ correspond to the complex eigenvalues of the matrix $P_{n}$. So we can now rewrite the last formula by integrating out the $x_{j a}$ 's, and by expressing the result in terms of the $z_{a}$ 's. This gives the standard form of the partition function

$$
Z_{3}=C_{3} \int \prod_{a=1}^{N}\left(d^{2} z_{a} w_{3}\left(z_{a}\right)\right) \prod_{b>a}^{N}\left|z_{b}-z_{a}\right|^{2}
$$

however with a non-standard weight function:

$$
w_{3}(z)=\int d^{2} x_{1} d^{2} x_{2} d^{2} x_{3} \delta^{(2)}\left(z-x_{1} x_{2} x_{3}\right) \mathrm{e}^{-\left(\left|x_{1}\right|^{2}+\left|x_{2}\right|^{2}+\left|x_{3}\right|^{2}\right)} .
$$

\subsection{The weight function}

It is easy to make the generalisation of the previous two subsections to the product (3.1) of any number of matrices $n$. Apart from the constants the only difference is that the weight function $w_{3}(z)$ has to be replaced by $w_{n}(z)$ :

$$
Z_{n}=C_{n} \int \prod_{a=1}^{N}\left(d^{2} z_{a} w_{n}\left(z_{a}\right)\right) \prod_{b<a}^{N}\left|z_{b}-z_{a}\right|^{2}, \quad C_{n}=\frac{1}{\left(N ! G[N+1] \pi^{N}\right)^{n}}=\left(C_{1}\right)^{n}
$$

that is given by:

$$
w_{n}(z)=\int \prod_{j=1}^{n} d^{2} x_{j} \delta^{(2)}\left(z-\prod_{j=1}^{n} x_{j}\right) \mathrm{e}^{-\sum_{j=1}^{n}\left|x_{j}\right|^{2}} .
$$

If one introduced a probabilistic normalisation to the weight function, the last equation could be interpreted in terms of the probability density function of a random variable obtained being a product of $n$ independent Gaussian complex random variables - very close to the spirit of our original problem. Of course things are more complicated since in addition to the weights we have also the repulsion coming from the Vandermonde determinants. The second observation is that the moments of the weight function factorise into $n$ independent Gaussian integrals

$$
\int d^{2} z|z|^{2 k} w_{n}(z)=\prod_{j=1}^{n} \int d^{2} x_{j}\left|x_{j}\right|^{2 k} \mathrm{e}^{-\left|x_{j}\right|^{2}}=\left(\int d^{2} x|x|^{2 k} \mathrm{e}^{-|x|^{2}}\right)^{n}=(\pi k !)^{n}
$$


since integrating out the delta constraint in (3.10) makes the $x_{j}$-integrals mutually independent.

One can find an integral representation of the weight function (3.10) by integrating out one variable, say $x_{n}=z /\left(x_{1} \ldots x_{n-1}\right)$, and getting rid of the delta function constraint. Equivalently it can be done using the formula (3.11) for $k=0$ in polar coordinates:

$$
r_{n} \rightarrow \frac{z}{r_{1} \cdots r_{n-1}}=\frac{r \exp [i \phi]}{r_{1} \cdots r_{n-1}}, n>1,
$$

where $r_{j}=\left|x_{j}\right|, r=|z|$. This leads to the following transformation:

$$
\begin{aligned}
& \int d^{2} z w_{n}(z)=\int d^{2} x_{1} \ldots d^{2} x_{n} \exp \left[-\sum_{j=1}^{n}\left|x_{j}\right|^{2}\right] \\
& =\int_{0}^{2 \pi} d \phi \int_{0}^{\infty} d r r(2 \pi)^{n-1} \int_{0}^{\infty} d r_{1} r_{1} \ldots d r_{n-1} r_{n-1} \frac{1}{\left(r_{1} \cdots r_{n-1}\right)^{2}} \exp \left[-\frac{r^{2}}{\left(r_{1} \cdots r_{n-1}\right)^{2}}-\sum_{j=1}^{n-1} r_{j}^{2}\right]
\end{aligned}
$$

with

$$
w_{n}(z)=(2 \pi)^{n-1} \int_{0}^{\infty} \frac{d r_{1} \ldots d r_{n-1}}{r_{1} \cdots r_{n-1}} \exp \left[-\frac{|z|^{2}}{\left(r_{1} \cdots r_{n-1}\right)^{2}}-\sum_{j=1}^{n-1} r_{j}^{2}\right], \quad n>1 .
$$

Obviously the weight given by an $(n-1)$-fold integral only depends on the modulus $|z|$. It immediately follows from (3.14) that

$$
w_{n+1}(z)=2 \pi \int_{0}^{\infty} \frac{d r}{r} w_{n}(z / r) \exp \left[-r^{2}\right], \quad n>0
$$

with $w_{1}(z)=\mathrm{e}^{-|z|^{2}}$ which is the Ginibre weight. This recursion relation can be solved for $w_{n+1}(z)$ by using the Mellin transform in the variable $R=|z|^{2}$. Defining $\Omega_{n}(R) \equiv w_{n}(\sqrt{R})$ we calculate the corresponding transform:

$$
M_{n}(s)=\int_{0}^{\infty} d R R^{s-1} \Omega_{n}(R) .
$$

The recursion relation (3.15) takes the form

$$
\Omega_{n+1}(R)=\pi \int_{0}^{\infty} \frac{d \rho}{\rho} \Omega_{n}(R / \rho) \mathrm{e}^{-\rho}
$$

and its Mellin transform factorises

$$
M_{n+1}(s)=\pi M_{n}(s) \Gamma(s),
$$

where $\Gamma(s)=\int_{0}^{\infty} d \rho \rho^{s-1} \mathrm{e}^{-\rho}$. Together with the initial condition

$$
M_{1}(s)=\int_{0}^{\infty} d R R^{s-1} \exp [-R]=\Gamma(s)
$$

we thus have

$$
M_{n}(s)=\pi^{n-1} \Gamma^{n}(s) .
$$

We can now write the inverse transform

$$
w_{n}(z)=\Omega_{n}\left(|z|^{2}\right)=\pi^{n-1} \frac{1}{2 \pi i} \int_{c-i \infty}^{c+i \infty} \Gamma^{n}(s)|z|^{-2 s} d s=\pi^{n-1} G_{0 n}^{n 0}\left(\left.\overline{\overrightarrow{0}}|| z\right|^{2}\right),
$$


where the symbol $G_{p q}^{n m}(\ldots \mid z)$ denotes Meijer's $G$-function [25], and $\overrightarrow{0}$ is a string of $n$-zeros. Only the subclass $G_{0 n}^{n 0}$ of these functions, corresponding to the inverse Mellin transform of $\Gamma^{n}(s)$, appears in this context. Note that our weight functions differ from the Meijer $G$-functions $G_{q q}^{q 0}$ appearing in the distribution of Fuss-Catalan numbers [4].

For $n=1$ this result reproduces by construction the weight for Ginibre matrices (or by inverse Mellin transform [25])

$$
w_{1}(z)=\exp \left[-|z|^{2}\right]=G_{01}^{10}\left(\left.\frac{-}{0}|| z\right|^{2}\right) .
$$

For $n=2$ the integral eq. (3.14) can be performed analytically, leading to a modified Bessel-function [6]:

$$
w_{2}(z)=2 \pi K_{0}(2|z|)=\pi G_{02}^{20}\left(\left.\overline{-}_{0,0}|| z\right|^{2}\right) .
$$

Alternatively one can use the connection of Meijer $G$-functions to special functions in [25]. For $n=3$ we either keep a single integral over the previous $K$-Bessel function, or we write the result explicitly in terms of the Meijer $G$-function:

$$
w_{3}(|z|)=4 \pi^{2} \int_{0}^{\infty} \frac{d r}{r} K_{0}(2|z| / r) \exp \left[-r^{2}\right]=\pi^{2} G_{03}^{30}\left(\left.{ }_{0,0,0}|| z\right|^{2}\right) .
$$

For an alternative representation in terms of hypergeometric functions and series of polygamma special functions we refer to [26] section $\mathrm{V}$ case $\mathrm{f}$ ).

\section{The orthogonal polynomial approach}

Since the partition function $Z_{n}$ (3.9) expressed in terms of the eigenvalues of the matrix $P_{n}$ has the standard form of the product of the weights times the absolute value square of the Vandermonde determinant we can use the standard technique of orthogonal polynomials in the complex plane to determine the $k$-point correlation functions [23. For recent reviews on non-Hermitian random matrix theory and its corresponding polynomials we refer to [18, 13]. We refer the interested reader to these references for details.

\subsection{Orthonormal polynomials and kernel}

From the fact that our weight $w_{n}(z)$ is angle-independent it immediately follows that the corresponding orthogonal polynomials are monic $p_{k}(z)=z^{k}$. Indeed, the integral

$$
\int d^{2} z w_{n}(z) z^{k} z^{* l}=\int_{0}^{2 \pi} d \phi \mathrm{e}^{i \phi(k-l)} \int_{0}^{\infty} d r r w(r) \equiv \delta_{k l} h_{k}^{(n)}
$$

gives zero for $k \neq l$. Using eq. (3.11) we immediately find that the squared norms are

$$
h_{k}^{(n)}=(\pi k !)^{n},
$$

so that the corresponding kernel of orthonormal polynomials reads

$$
K_{N}^{(n)}\left(z_{i}, z_{j}\right)=\sqrt{w\left(z_{i}\right) w\left(z_{j}\right)} \sum_{k=0}^{N-1} \frac{1}{(\pi k !)^{n}}\left(z_{i} z_{j}^{*}\right)^{k} .
$$

In view of the results later in section 6.3 we call this a truncated hypergeometric kernel, just as in the Ginibre case $n=1$ this is a truncated exponential. 
Following [23] this kernel determines the $k$-point correlation functions:

$$
R_{k}^{(n)}\left(z_{1}, \ldots, z_{k}\right)=\operatorname{det}_{1 \leq i, j, \leq k}\left[K_{N}^{(n)}\left(z_{i}, z_{j}\right)\right] .
$$

In particular, the one-point correlation function or spectral density is $R_{1}^{(n)}(z)=K_{N}^{(n)}(z, z)$, the twopoint correlation function is $R_{2}^{(n)}(z, u)=K_{N}^{(n)}(z, z) K_{N}^{(n)}(u, u)-K_{N}^{(n)}(z, u) K_{N}^{(n)}(u, z)$ etc. We are now going to discuss these in the large- $N$ limit in the next section.

\section{Eigenvalue density at large- $N$}

In this section we study the eigenvalue density for large- $N$ (and large argument). The purpose of that is two-fold, as we will not only find the mean or macroscopic density after a suitable rescaling. The expression for large but finite- $N$ that we will derive below enables us to identify the different regions in the complex plane where we can take different microscopic limits that describe the fluctuations in that region.

We begin with the definition of the spectral density or one-point correlation function in terms of the kernel:

$$
R_{1}^{(n)}(z)=K_{N}^{(n)}(z, z)=w_{n}(z) \sum_{k=0}^{N-1} \frac{|z|^{2 k}}{(\pi k !)^{n}} \equiv w_{n}(z) T_{n}\left(|z|^{2}, N\right) .
$$

We note that according to its definition eq. (2.5) it is normalised to the number of eigenvalues, $\int d^{2} z R_{1}^{(n)}(z)=N$, rather than to unity.

Now we are going to determine the behaviour of $R_{1}^{(n)}(z)$ for large $N$ and for large $|z|$. The asymptotic large- $|z|$ behaviour of the weight $w_{n}(z)$ can be taken from [27]. It is rederived here for completeness in Appendix B (see equation (B.5)). The large- $N$ behaviour of the truncated sum $T_{n}(x, N)$ for $x=|z|^{2}$ of order $N^{n}$ was already studied in [28], and we give some details of the derivation in Appendix $\mathrm{C}$ (see equation (C.6)). Putting together (B.5) and (C.6) we obtain the following behaviour for large- $N$ and for $|z| \lesssim N^{n / 2}$ :

$$
R_{1}^{(n)}(z) \approx \frac{|z|^{\frac{2}{n}-2}}{n \pi} \frac{1}{2} \operatorname{erfc}\left(\frac{\sqrt{n}\left(|z|^{2 / n}-N\right)}{\sqrt{2}|z|^{1 / n}}\right) .
$$

It is convenient to recast this result into a rescaled density with compact support that is normalised to unity. Using the rescaled variable $w=z N^{-n / 2}$ we define the following density, for which the radius of the eigenvalue support approaches one for $N \rightarrow \infty$ :

$$
\begin{aligned}
\rho_{n}(w) & \equiv \lim _{N \gg 1} N^{n-1} R_{1}^{(n)}\left(N^{n / 2} w\right)=\frac{|w|^{\frac{2}{n}}-2}{n \pi} \frac{1}{2} \operatorname{erfc}\left(\frac{\sqrt{n N}\left(|w|^{2 / n}-1\right)}{\sqrt{2}|w|^{1 / n}}\right) \\
& =\frac{|w|^{\frac{2}{n}-2}}{n \pi} \frac{1}{2} \operatorname{erfc}\left(\sqrt{\frac{2 N}{n}}(|w|-1)\right) .
\end{aligned}
$$

The factor $N^{n}$ in the first step comes from the rescaling of the volume element $d^{2} z=N^{n} d^{2} w$, and the additional factor $N^{-1}$ from the normalisation to unity. In the second step we have simplified $\rho_{n}(w)$ by Taylor expanding the argument around unity, $|w|=(2 / n)(|w|-1)+O\left((|w|-1)^{2}\right)$. This is because the complementary error function changes only in a narrow strip around the unit circle $|w|=1$, of a width proportional to $1 / \sqrt{N}$. So far we have derived a finite size form of the eigenvalue distribution of the product of $n$ independent Ginibre matrices. This form was known for $n=1$ [16, 17] and $n=2$ 
[15]. It was conjectured to hold for any $n$ and tested numerically in [9] except that the dependence of the coefficient inside the erfc-function on $n$ was unknown. We have filled this gap here by deriving eq. (5.3) for any $n$. We see that the width of the crossover region around the edge $|w|=1$ is proportional to the square root of the number of multiplied matrices $\sqrt{n}$.

It is instructive to compare this result with the limiting density for large- $N$ of the $n$-th power of a single Ginibre matrix. This density is derived in Appendix $\mathrm{D}$ and is given by exactly the same distribution, however with a different dependence on $n$ :

$$
\tilde{\rho}_{n}(w)=\frac{|w|^{\frac{2}{n}}-2}{n \pi} \frac{1}{2} \operatorname{erfc}\left(\frac{\sqrt{2 N}}{n}(|w|-1)\right) .
$$

Here, the width of the crossover region is proportional to $n$ and not to $\sqrt{n}$. In a sense, the finite size corrections are stronger for the $n$-th power than for the product of $n$ independent Ginibre matrices.

Actually the same seems to be true for a broader class of matrices called isotropic [29]. Isotropic matrices are known to have the property that the product of iid matrices has the same limiting distribution as the corresponding power of a single one, as was discussed in [29]. For this class the finite size formula is not known but numerically one also observes that the product of independent matrices converges faster to the limiting distribution than does the corresponding power [29].

We close this section by rederiving the mean or macroscopic density in the limit $N \rightarrow \infty$. It is obtained by taking the limit $N \rightarrow \infty$ of (5.3):

$$
\rho_{\text {macro }}^{(n)}(w) \equiv \lim _{N \rightarrow \infty} \rho_{n}(w)=\frac{|w|^{\frac{2}{n}-2}}{n \pi} \Theta(1-|w|),
$$

where $\Theta(x)$ is the Heaviside step function. This result was derived in [7] using planar diagrams and rigorously in [8, 10]. We also refer to $[8]$ for an extensive list of references regarding the mathematical literature on products of random matrices.

\section{Microscopic large- $N$ limits and universality}

\subsection{Universal edge limit}

In this subsection we analyse the fluctuations of the eigenvalues around the edge of the spectrum. We will only consider the density itself, higher order correlation functions can be dealt with analogously. Our starting point is the density at large- $N$ and large- $|z|$, eq. (5.2). As was already mentioned in the previous section the complementary error-function rapidly vanishes for $|z|>N^{n / 2}$, on a strip of width $1 / \sqrt{N}$. We therefore introduce a new scaling variable $\xi$ :

$$
z=N^{n / 2}(1+\xi \sqrt{n / N}) \mathrm{e}^{i \phi} .
$$

The additional rescaling with $\sqrt{n}$ is made in order to normalise properly. We can therefore define the microscopic density at the edge by

$$
\lim _{N \rightarrow \infty} \rho_{e d g e}^{(n)}(\xi) \equiv \lim _{N \rightarrow \infty} n N^{n-1} R_{1}^{(n)}\left(z=N^{n / 2}(1+\xi \sqrt{n / N}) \mathrm{e}^{i \phi}\right)=\frac{1}{2 \pi} \operatorname{erfc}(\sqrt{2} \xi),
$$

where for each real dimension the density $R_{1}$ has to be rescaled by $\sqrt{n} N^{n / 2}$. The resulting expression agrees with the $n=1$ result for the Ginibre ensemble derived in [16, 17] (see also [23]). For $n=2$ the same result was previously derived in [15. We have shown here that the result holds true for any fixed $n$ and is thus universal. This behaviour was already observed numerically in [9]. 


\subsection{Universal bulk limit}

In this subsection we discuss the behaviour of the $k$-point correlation functions $R_{k}^{(n)}\left(z_{1}, \ldots, z_{k}\right)$ defined in (4.4), in the limit $N \rightarrow \infty$ for the following scale separation $1 \ll\left|z_{k}\right| \lesssim N^{n / 2}$. By comparing to eq. (5.2) this separation will guarantee that we are neither close to the origin to be discussed later, nor to the edge limit discussed above.

In principle the microscopic bulk limit is obtained in three steps. We would first have to rescale $z=N^{n / 2} w$ in order to get a compact support. In the second step we would zoom into the fluctuations which are in this case of the order $1 / N^{n / 2}, w=v / N^{n / 2}$. In the last step we would unfold the variables in order to get a locally flat density, with a mean level spacing of unity. Because of the macroscopic density being given by

$$
\rho_{\text {macro }}^{(n)}(w)=\frac{|w|^{\frac{2}{n}-2}}{n \pi} \Theta(1-|w|),
$$

the unfolding would read $\xi=\sqrt{n} v^{1 / n}$. Because the rescalings from the first two steps compensate each other here, we can do all steps in one. Our microscopic scaling limit is thus given by the change of variables

$$
1 \ll\left|\xi=\sqrt{n} z^{1 / n}\right| \lesssim \sqrt{N}
$$

which will become $N$-independent in the large- $N$ limit. Note that in the variable $z^{1 / n}$ the fluctuations are again of order $1 / \sqrt{N}$ as in the Ginibre ensemble. The change of variables for the $k$-point densities can be best seen by considering the two-dimensional differential

$$
d^{2} \xi=n^{-1}|z|^{\frac{2}{n}-2} d^{2} z
$$

We thus obtain for the microscopic density

$$
\widehat{R}_{1}^{(n)}(\xi) \equiv n|z|^{2-\frac{2}{n}} R_{1}^{(n)}\left(z=(\xi / \sqrt{n})^{n}\right)=n|z|^{2-\frac{2}{n}} \frac{1}{n \pi}|z|^{\frac{2}{n}-2}=\frac{1}{\pi},
$$

which is constant on a disk of radius $\sqrt{N}$ and zero otherwise. So indeed the eigenvalues are uniformly distributed in the $\xi$-plane for large $|\xi|$ inside the support. More generally, the microscopic $k$-point correlation functions are defined as

$$
\widehat{R}_{k}^{(n)}\left(\xi_{1}, \ldots, \xi_{k}\right) \equiv n^{k}\left|z_{1} \ldots z_{k}\right|^{2-2 / n} R_{k}^{(n)}\left(z_{1}, \ldots, z_{k}\right),
$$

where $z_{j}=\left(\xi_{j} / \sqrt{n}\right)^{n}$ on the right hand side, from eq. (6.4). All $k$-point correlation functions $\widehat{R}_{k}^{(n)}\left(\xi_{1}, \ldots, \xi_{k}\right)$ can be expressed in terms of a redefined kernel

$$
\widehat{R}_{k}^{(n)}\left(\xi_{1}, \ldots, \xi_{k}\right)=\operatorname{det}_{1 \leq i, j, \leq k}\left[\widehat{K}_{N}^{(n)}\left(\xi_{i}, \xi_{j}\right)\right],
$$

that is given by

$$
\widehat{K}_{N}^{(n)}\left(\xi_{i}, \xi_{j}\right)=n\left|z_{i} z_{j}\right|^{1-1 / n} K_{N}^{(n)}\left(z_{i}, z_{j}\right)=n^{2-n}\left|\xi_{i} \xi_{j}\right|^{n-1} K_{N}^{(n)}\left(\left(\xi_{i} / \sqrt{n}\right)^{n},\left(\xi_{j} / \sqrt{n}\right)^{n}\right) .
$$

In the limit $N \rightarrow \infty$ and $1 \ll\left|\xi_{j}\right| \lesssim \sqrt{N}$ the limiting kernel in the bulk is given by

$$
\widehat{K}_{b u l k}^{(n)}\left(\xi_{i}, \xi_{j}\right) \equiv \lim _{N \rightarrow \infty ;\left|\xi_{i, j}\right| \gg 1} n^{2-n}\left|\xi_{i} \xi_{j}\right|^{n-1} \sqrt{w_{n}\left(\left(\xi_{i} / \sqrt{n}\right)^{n}\right) w_{n}\left(\left(\xi_{j} / \sqrt{n}\right)^{n}\right)} T_{n}\left(\left(\xi_{i} \xi_{j}^{*} / n\right)^{n}\right)
$$

where we have for asymptotically large argument (B.5)

$$
\lim _{|\xi| \gg 1} w_{n}\left((\xi / \sqrt{n})^{n}\right)=\frac{1}{\sqrt{n}}\left(2 \pi^{3} n\right)^{(n-1) / 2}|\xi|^{1-n} \mathrm{e}^{-|\xi|^{2}}
$$


and for asymptotically large argument and large- $N$ (C.6)

$$
\lim _{N \rightarrow \infty ;|\xi| \gg 1} T_{n}\left(\left(\xi_{i} \xi_{j}^{*} / n\right)^{n}\right)=\frac{1}{\pi \sqrt{n}}\left(2 \pi^{3} / n\right)^{(1-n) / 2}\left(\xi_{i} \xi_{j}^{*}\right)^{(1-n) / 2} \exp \left[\xi_{i} \xi_{j}^{*}\right] .
$$

Putting all together we obtain

$$
\widehat{K}_{b u l k}^{(n)}\left(\xi_{i}, \xi_{j}\right)=\frac{1}{\pi}\left(\frac{\xi_{i} \xi_{j}^{*}}{\left|\xi_{i} \xi_{j}\right|}\right)^{(1-n) / 2} \exp \left[-\frac{1}{2}\left(\left|\xi_{i}\right|^{2}+\left|\xi_{j}\right|^{2}+\xi_{i} \xi_{j}^{*}\right)\right] .
$$

We see that the kernel is Hermitian $\widehat{K}^{(n)}\left(\xi_{i}, \xi_{j}\right)^{*}=\widehat{K}^{(n)}\left(\xi_{j}, \xi_{i}\right)$. It is equal to the kernel of the Ginibre ensemble multiplied by powers of the phase factors $\mathrm{e}^{i \phi_{j}}=\xi_{j} /\left|\xi_{j}\right|$. The kernel (6.13) is unitarily equivalent to the kernel of the Ginibre ensemble. When one calculates the correlation functions $\widehat{R}_{k}^{(n)}\left(\xi_{1}, \ldots, \xi_{k}\right)$ (6.8) all phase factors cancel and one obtains exactly the same $k$-point correlation functions as for the Ginibre ensemble with $n=1$. Therefore all correlation functions of the product matrix $P_{n}$ in the bulk limit are universal. In particular the two-point correlation function is given by

$$
\widehat{R}_{b u l k}^{(n)}\left(\xi_{1}, \xi_{2}\right)=\frac{1}{\pi^{2}}\left(1-\mathrm{e}^{-\left|\xi_{1}-\xi_{2}\right|^{2}}\right),
$$

which describes correlations of eigenvalues at distances $\left|\xi_{1}-\xi_{2}\right|$ of order unity.

\subsection{Microscopic origin limit: $\mathrm{n}$ classes}

In this subsection we investigate the remaining region where a non-trivial microscopic limit can be obtained, that is the vicinity of the origin. While the microscopic bulk and edge limits yield universal results for arbitrary fixed $n$, at the origin we obtain a different kernel for each $n$. For $n=1$ it coincides with the bulk limit whereas for $n=2$ we obtain a kernel containing Bessel- $K$ and Bessel- $I$ functions which was known based on the work [6], see also [14 for more details.

If we look back at the previous two limits the edge correlations were found for $|z| \approx N^{n / 2}$ or $|w| \approx 1$ in rescaled variables $z=N^{n / 2} w$, with fluctuations of order $1 / \sqrt{N}$. The bulk limit was obtained by considering $1 \ll|z| \lesssim N^{n / 2}$ or $0<|w|<1$, with fluctuations again of order $1 / \sqrt{N}$ in terms of the rescaled variable $\xi=\sqrt{n} z^{1 / n}$. Here we will take the limit $N \rightarrow \infty$ keeping $|z|=\mathcal{O}(1)$, or $|w| \approx N^{-n / 2}$. Because of that the scaling is very simple in $z$ : the weight $w_{n}(z)$ which is $N$-independent remains unchanged, and the sum inside the kernel eq. (5.1) simply has to be extended to infinity:

$$
K_{\text {origin }}^{(n)}\left(z_{i}, z_{j}\right) \equiv \lim _{N \rightarrow \infty} K_{N}^{(n)}\left(z_{i}, z_{j}\right)=\sqrt{w_{n}\left(z_{i}\right) w_{n}\left(z_{j}\right)}{ }_{0} F_{n-1}\left(-; \overrightarrow{1} ; z_{i} z_{j}^{*}\right) \pi^{-n}, n>1
$$

Here we have used that the infinite sum eq. (C.7) is related to a generalised hypergeometric function,

$$
T_{n}(x)=\sum_{k=0}^{\infty} \frac{x^{k}}{(\pi k !)^{n}}=\frac{1}{\pi^{n}}{ }_{0} F_{n-1}(-; \overrightarrow{1} ; x)
$$

for $n>1$. The vector $\overrightarrow{1}$ contains $n-1$ elements of unity. For $n=1$ we simply have

$$
T_{1}(x)=\sum_{k=0}^{\infty} \frac{x^{k}}{\pi k !}=\frac{1}{\pi} \exp [x]
$$

It cancels the exponent $\exp \left[-|z|^{2}\right]$ coming from the weight and thus leads to a constant density. 
For $n=2$ the result can also be expressed in terms of elementary functions, due to

$$
T_{2}(x)=\sum_{k=0}^{\infty} \frac{x^{k}}{(\pi k !)^{2}}=\frac{1}{\pi^{2}} I_{0}(2 \sqrt{x}) .
$$

This leads to the known kernel (see e.g. [14])

$$
K_{\text {origin }}^{(n=2)}\left(z_{i}, z_{j}\right)=\frac{2}{\pi} \sqrt{K_{0}\left(2\left|z_{i}\right|\right) K_{0}\left(2\left|z_{j}\right|\right)} I_{0}\left(2\left|z_{i} z_{j}\right|^{\frac{1}{2}}\right)
$$

in terms of Bessel functions, with the density $\rho_{\text {origin }}^{(n=2)}(z)=\frac{2}{\pi} K_{0}(2|z|) I_{0}(2|z|)$. For $n \geq 3$ our kernel eq. (6.15) seems to be new. Although other random matrix ensembles with kernels containing hypergeometric functions exist, we are not aware of any example containing ${ }_{0} F_{n-1}$.

\section{Conclusions and outlook}

In this paper we have investigated the eigenvalue correlations for the product of $n$ independent nonHermitian random matrices. Explicit results were given for any finite matrix size $N$ for all density correlation functions in terms of the determinant of a kernel of orthogonal polynomials in the complex plane. The corresponding weight functions were found to be so-called Meijer $G$-functions depending on $n$, whereas the joint probability distribution function remained the absolute value squared of the Vandermonde determinant which is standard for this symmetry class.

So far such results were only known for up to $n=2$ (in fact for rectangular matrices with an elliptic law). In the large- $N$ limit we investigated three different microscopic limits where the local fluctuations were zoomed into: the microscopic edge, bulk and origin limit. In the former two cases we found a complete agreement with the Ginibre ensemble of a single matrix $n=1$, after unfolding. The edge and bulk limit are thus universal, as it was conjectured earlier for the behaviour at the edge depending on the complementary error function. In contrast at the origin each new matrix in the product adds a new class of correlation functions, given by an $n$-dependent hypergeometric kernel together with the Meijer $G$-function from the weight.

Several generalisations of the results presented here are conceivable. First of all it should be possible to consider the product of rectangular matrices instead. We expect that the results for the edge and the bulk remain unchanged, whereas the origin limit will be generalised as it is already known for $n=2$. Matrices from other symmetry classes could be considered, such as real quaternionic non-self dual or real asymmetric matrices. Again we expect the edge and bulk behaviour to be unchanged, with local microscopic modifications along the real and imaginary axis as well as at the origin. Finally also for each matrix more general distributions than the Gaussian Ginibre distribution could be feasible. In particular it would be interesting to know if the microscopic properties we computed continue to hold for the product of non-Hermitian Wigner matrices with iid but non-Gaussian entries. Several of these projects are currently under way.

Acknowledgments: We would like to thank Eugene Strahov for useful discussions. We acknowledge partial support by the Polish Ministry of Science Grant No. N N202 229137 (2009-2012) and by the Grant DEC-2011/02/A/ST1/00119 of the National Centre of Science (Z.B.), as well as by the SFB | TR12 "Symmetries and Universality in Mesoscopic Systems" of the German research council DFG (G.A.). 


\section{A Computation of the Jacobian}

We now calculate the Jacobi matrix for the change of variables from $X$ to $(U, T, \Lambda)$. In order to determine the relation between the infinitesimal elements (one-forms) we differentiate both sides of (3.5). We obtain

$$
\begin{aligned}
d X & =\left(\begin{array}{ccc}
0 & d X_{1} & 0 \\
0 & 0 & d X_{2} \\
d X_{3} & 0 & 0
\end{array}\right) \\
& =\left(\begin{array}{ccc}
U_{1} & 0 & 0 \\
0 & U_{2} & 0 \\
0 & 0 & U_{3}
\end{array}\right)\left(\begin{array}{ccc}
0 & d Y_{1} & 0 \\
0 & 0 & d Y_{2} \\
d Y_{3} & 0 & 0
\end{array}\right)\left(\begin{array}{ccc}
U_{1}^{-1} & 0 & 0 \\
0 & U_{2}^{-1} & 0 \\
0 & 0 & U_{3}^{-1}
\end{array}\right)
\end{aligned}
$$

where

$$
d Y_{j}=d \Lambda_{j}+d T_{j}+d M_{j}
$$

and

$$
\begin{aligned}
& \left(d M_{1}\right)_{a b}=\left(d A_{1}\right)_{a b} x_{1 b}-x_{1 a}\left(d A_{2}\right)_{a b}+\sum_{c<b}\left(d A_{1}\right)_{a c}\left(T_{1}\right)_{c b}-\sum_{d>a}\left(T_{1}\right)_{a d}\left(d A_{2}\right)_{d b} \\
& \left(d M_{2}\right)_{a b}=\left(d A_{2}\right)_{a b} x_{2 b}-x_{2 a}\left(d A_{3}\right)_{a b}+\sum_{c<b}\left(d A_{2}\right)_{a c}\left(T_{2}\right)_{c b}-\sum_{d>a}\left(T_{2}\right)_{a d}\left(d A_{3}\right)_{d b} \\
& \left(d M_{3}\right)_{a b}=\left(d A_{3}\right)_{a b} x_{3 b}-x_{3 a}\left(d A_{1}\right)_{a b}+\sum_{c<b}\left(d A_{3}\right)_{a c}\left(T_{3}\right)_{c b}-\sum_{d>a}\left(T_{3}\right)_{a d}\left(d A_{1}\right)_{d b}
\end{aligned}
$$

The $d A_{j}=U_{j}^{-1} d U_{j}$ are anti-Hermitian matrices with zeros on the diagonal. One can choose $\left(d A_{j}\right)_{a b}$ and $\left(d A_{j}\right)_{a b}^{*}$ in the upper triangle $(a>b)$ to be independent infinitesimal elements of $d A_{j}$ (or more precisely - independent one-forms). The elements $\left(d A_{j}\right)_{a b}$ in the lower triangle $(a<b)$, can be expressed by those in the upper one: $\left(d A_{j}\right)_{a b}=-\left(d A_{j}\right)_{b a}^{*}$. The diagonal elements of $d A_{j}$ are zero since $U_{j}$ are restricted to $U(N) / U(1)^{N}$.

Now we are ready to calculate the Jacobian. Let us do it gradually. First observe that the Jacobian for the change of variables $d X=U d Y U^{-1}$ eq. (A.1) equals one since the matrix $U$ is unitary. So we have $|D X|=|D Y|$. Now we can calculate the Jacobian for the change from $d Y$ to $(d \Lambda, d T, d M)$ in eq. (A.2). To this end let us write down (A.2) in an explicit index notation

$$
\begin{array}{lrll}
\left(d Y_{j}\right)_{a b}=d\left(\Lambda_{j}\right)_{a b} & + & \left(d M_{j}\right)_{a b} & \text { for } a=b, \\
\left(d Y_{j}\right)_{a b}= & d\left(T_{j}\right)_{a b}+ & \left(d M_{j}\right)_{a b} & \text { for } a<b, \\
\left(d Y_{j}\right)_{a b}= & & \left(d M_{j}\right)_{a b} & \text { for } a>b .
\end{array}
$$

There are also corresponding equations for the complex conjugates. The first line, for $a=b$, could be alternatively written as $\left(d Y_{j}\right)_{a a}=d x_{j a}+\left(d M_{j}\right)_{a a}$ since $d\left(\Lambda_{j}\right)_{a b}=\delta_{a b} d x_{j a}$, or in shorthand notation $d \Lambda=d x$. As one can see from (A.4) the Jacobi matrix for the linear transformation from the $d Y$-basis to the $(d x, d T, d M)$-basis is upper triangular and has a Jacobian equal one, so we have

$$
|D X|=|D Y|=|D x||D T||D M| .
$$

The measure $D M$ in the last equation is a product of one-forms $\left(d M_{j}\right)_{a b}$ for $a>b$. They can be expressed as a linear combination of the one-forms $d A$ 's in eq. A.3):

$$
|D X|=|D x||D T||D A|\left|\frac{\partial M}{\partial A}\right| \text {. }
$$


Let us calculate the Jacobian $|J|=|\partial M / \partial A|$ for this transformation (A.3). It has a specific algebraic structure that imposes a certain ordering of the indices that appear in (A.3). In fact this ordering implies that the Jacobi matrix can be written as an upper triangular block matrix. To see this let us first recall that we are considering only the sector $a>b$ corresponding to the lowest line of (A.4). Second of all, let us note that as a result of the $T_{j}$ 's being strictly upper triangular matrices we have further inequalities for the indices in (A.3):

$$
a>b>c \text { and } d>a>b .
$$

An inspection of the indices of the one-forms appearing in (A.3), $\left(d M_{j}\right)_{a b}(a>b),\left(d A_{j}\right)_{a c}(a>c)$, $\left(d A_{j}\right)_{d b}(d>b)$, shows that they are all indexed by ordered pairs of indices whose first index is larger than the second one. It is convenient to introduce an increasing ordering in the set of such ordered pairs. A pair $a b$ is said to be less than a pair $c d$ (and denoted by $a b<c d$ ) if $a>c$ or if $a=c$ and $b<d$. This choice of ordering does not look very intuitive but it is convenient for our purposes. For example for $N=4$ there are six ordered pairs and they are ordered as follows $41<42<43<31<32<21$. We can now introduce a single index $\alpha=1, \ldots, 6$ that preserves the increasing ordering of the pairs: $41 \rightarrow 1,42 \rightarrow 2,42 \rightarrow 3,31 \rightarrow 4,31 \rightarrow 5,21 \rightarrow 6$. Of course, we can do this for any $N$. Using the index $\alpha$ we can concisely write equations (A.3) as

$$
\left(d M_{j}\right)_{\alpha}=\sum_{j^{\prime} \alpha^{\prime}}\left(J_{j j^{\prime}}\right)_{\alpha \alpha^{\prime}}\left(d A_{k}\right)_{\alpha^{\prime}} .
$$

Now we want to argue that the Jacobi matrix is upper block triangular, that is $\left(J_{j j^{\prime}}\right)_{\alpha \alpha^{\prime}}=0$ for all $\alpha>\alpha^{\prime}$. Clearly the matrix has diagonal blocks $\alpha=\alpha^{\prime}$ (A.3). It also has off-diagonal blocks coming from the sums on the right-hand side of (A.3). We now show that the terms in the sums contribute to the upper triangle $\alpha<\alpha^{\prime}$. Indeed, as follows from the inequalities (A.7) the pairs of indices $a b$ of $\left(d M_{j}\right)_{a b}$ 's on the left hand side of (A.3) are smaller (in the sense defined above: $a b<a c$ and $a b<d b$ ) than the corresponding pairs $a c$ and $d b$ of $\left(d A_{j}\right)_{a c}$ and $\left(d A_{j}\right)_{d b}$ in the sums on the right hand side of (A.3). This is equivalent to saying that the sums run over $\alpha^{\prime \prime}$ 's such that $\alpha<\alpha^{\prime}$. There are no terms for $\alpha>\alpha^{\prime}$ on the right hand side of (A.3) and thus the Jacobi matrix (A.8) $\left(J_{j j^{\prime}}\right)_{\alpha \alpha^{\prime}}=0$ for $\alpha>\alpha^{\prime}$. Since the Jacobi matrix $\left(J_{j j^{\prime}}\right)_{\alpha \alpha^{\prime}}$ is upper block triangular, its Jacobi determinant $|J|$ is identical to the determinant of the corresponding block diagonal matrix $\left(\widehat{J}_{j j^{\prime}}\right)_{\alpha \alpha^{\prime}}$ that has the same diagonal blocks: $|J|=|\widehat{J}|$. In this way we have reduced the calculation of the determinant $|\partial M / \partial A|$ to the calculation of the determinant $|\partial \widehat{M} / \partial A|$ of the block diagonal matrix $\widehat{J}$ corresponding to the transformation obtained from (A.3) by skipping upper-triangular terms, that is the sums:

$$
\begin{aligned}
& \left(d \widehat{M}_{1}\right)_{a b}=\left(d A_{1}\right)_{a b} x_{1 b}-x_{1 a}\left(d A_{2}\right)_{a b}, \\
& \left(d \widehat{M}_{2}\right)_{a b}=\left(d A_{2}\right)_{a b} x_{2 b}-x_{2 a}\left(d A_{3}\right)_{a b}, \\
& \left(d \widehat{M}_{3}\right)_{a b}=\left(d A_{3}\right)_{a b} x_{3 b}-x_{3 a}\left(d A_{1}\right)_{a b} .
\end{aligned}
$$

The Jacobian can be calculated by the use of the external (wedge) product of one-forms $\left(d \widehat{M}_{j}\right)_{a b}$. Let us first calculate it for a given pair $a b$. Using (A.3) we have:

$$
\begin{aligned}
& \left(d \widehat{M}_{1}\right)_{a b} \wedge\left(d \widehat{M}_{2}\right)_{a b} \wedge\left(d \widehat{M}_{3}\right)_{a b}= \\
& \left(d A_{1}\right)_{a b} \wedge\left(d A_{2}\right)_{a b} \wedge\left(d A_{3}\right)_{a b}\left(x_{1 b} x_{2 b} x_{3 b}-x_{1 a} x_{2 a} x_{3 a}\right) .
\end{aligned}
$$

Taking also into account the corresponding independent equations for the complex conjugates, we 
obtain a transformation of the 6 -form in the whole $a b$ sector :

$$
\begin{aligned}
& \left(d \widehat{M}_{1}\right)_{a b} \wedge\left(d \widehat{M}_{2}\right)_{a b} \wedge\left(d \widehat{M}_{3}\right)_{a b} \wedge\left(d \widehat{M}_{3}\right)_{a b}^{*} \wedge\left(d \widehat{M}_{2}\right)_{a b}^{*} \wedge\left(d \widehat{M}_{1}\right)_{a b}^{*}= \\
& \left(d A_{1}\right)_{a b} \wedge\left(d A_{2}\right)_{a b} \wedge\left(d A_{3}\right)_{a b} \wedge\left(d A_{3}\right)_{a b}^{*} \wedge\left(d A_{2}\right)_{a b}^{*} \wedge\left(d A_{1}\right)_{a b}^{*} \\
& \times\left|x_{1 b} x_{2 b} x_{3 b}-x_{1 a} x_{2 a} x_{3 a}\right|^{2} .
\end{aligned}
$$

We repeat this calculation independently for all sectors $a>b$ and eventually obtain the volume form:

$$
|D M|=|J(x)||D A|=\prod_{a>b}^{N}\left|x_{1 b} x_{2 b} x_{3 b}-x_{1 a} x_{2 a} x_{3 a}\right|^{2}|D A|,
$$

and

$$
|D X|=|J(x)||D x||D T||D A| .
$$

We are ready to write down the partition function (3.2) in new variables, for general $n$. The Gaussian weight function (3.2) assumes a form:

$$
\mathrm{e}^{-\operatorname{Tr} \sum_{j=1}^{n} X_{j}^{\dagger} X_{j}}=\mathrm{e}^{-\sum_{j=1}^{n} \sum_{a=1}^{N}\left|x_{j a}\right|^{2}} \mathrm{e}^{-\sum_{j=1}^{n} \sum_{a>b}^{N}\left|\left(T_{j}\right)_{a b}\right|^{2}}
$$

that is independent of $d A_{j}$ 's and thus of $d U_{j}$ 's: $d A_{j}=U_{j}^{-1} d U_{j}$. So we can integrate out the $d U$ variables. This integration gives a constant equal to the volume of the coset $U(N) / U(1)^{N}$. Also the integration over the $\left(T_{j}\right)_{a b}$ 's can be done since it is an independent Gaussian integral. By comparing to Ginibre we obtain for each $j$

$$
\int\left|D U_{j}\right| \int\left|D T_{j}\right| \exp \left[-\operatorname{Tr} T_{j}^{\dagger} T_{j}\right]=1 /\left(N ! G[N+1] \pi^{N}\right)=C_{1} .
$$

Denoting by $C_{n}$ the total constant factor coming from the integration over all $d U_{j}$ 's and $d T_{j}$ 's, we have $C_{n}=\left(C_{1}\right)^{n}$. What remains is an integral over the $d z$ 's as was claimed in section 3 ;

$$
Z_{n}=C_{n} \int \prod_{j=1}^{n} \prod_{a=1}^{N} d^{2} x_{j a} \prod_{a>b}^{N}\left|x_{1 b} x_{2 b} x_{3 b}-x_{1 a} x_{2 a} x_{3 a}\right|^{2} \mathrm{e}^{-\sum_{j=1}^{n} \sum_{a=1}^{N}\left|x_{j a}\right|^{2}} .
$$

\section{B Asymptotic of the weight function}

In order to make our paper self contained we compute here the leading order asymptotic behaviour of the weights $w_{n}(z)$ for $|z| \rightarrow \infty$ using the saddle point method. The result can also be found in Theorem 2 in reference [27].

We use the multidimensional representation of the weights (3.14):

$$
w_{n}(z)=(2 \pi)^{n-1} \int \prod_{j=1}^{n-1}\left(d r_{j} / r_{j}\right) \mathrm{e}^{-S}, \quad n>1
$$

where

$$
S=\frac{|z|^{2}}{\left(r_{1} \ldots r_{n-1}\right)^{2}}+\sum_{j=1}^{n-1} r_{j}^{2}
$$

The saddle point equation

$$
\frac{\partial S}{\partial r_{j}}=\frac{-2}{r_{j}} \frac{|z|^{2}}{\left(r_{1} \ldots r_{n-1}\right)^{2}}+2 r_{j}=0
$$


has a symmetric solution $r_{1}=r_{2}=\ldots r_{n-1} \equiv r_{*}=|z|^{1 / n}$. The equation has no other solutions, so the symmetric solution is not only a local minimum of $S$ but also the global one. At the minimum the function $S$ assumes the value $S_{*}=n|z|^{2 / n}$. The Hessian $H_{i j}=\frac{\partial^{2} S}{\partial r_{i} \partial r_{j}}=\frac{\left(4+2 \delta_{i j}\right)|z|^{2}}{r_{i} r_{j}\left(r_{1} \ldots r_{n-1}\right)^{2}}+2 \delta_{i j}$ takes the following values at the minimum $r_{*}: H_{* i j}=4$ for $i \neq j$ and $H_{* i j}=8$ for $i=j$. The determinant of the Hessian is $\operatorname{det}\left[H_{*}\right]=n 4^{n-1}$. So we can write an explicit formula for the leading order asymptotic behaviour of $w_{n}(z)$ for large $z$ as

$$
w_{n}(z) \sim\left(\frac{2 \pi}{r_{*}}\right)^{n-1} \frac{(2 \pi)^{(n-1) / 2}}{\left(\operatorname{det} H_{*}\right)^{1 / 2}} \mathrm{e}^{-S_{*}}
$$

that gives

$$
w_{n}(z) \sim \frac{1}{\sqrt{n}}\left(2 \pi^{3}\right)^{(n-1) / 2}|z|^{(1-n) / n} \mathrm{e}^{-n|z|^{2 / n}},
$$

which agrees with [27] where also the error terms are computed. In particular for $n=2,3$ we have

$$
w_{2}(z) \sim \pi^{3 / 2} z^{-1 / 2} \mathrm{e}^{-2|z|}, w_{3}(z) \sim \frac{2 \pi^{3}}{\sqrt{3}}|z|^{-2 / 3} \mathrm{e}^{-3|z|^{2 / 3}},
$$

where the first result $n=2$ agrees with the known asymptotic of $K_{0}(2|z|)$ from eq. (3.23) [25]. In fact eq. (B.5) also holds for $n=1$ where $w_{n}(z) \sim \exp \left[-|z|^{2}\right]$ is exact.

The asymptotic formula (B.5) is consistent with the recurrence relation (3.15) in the sense that when one inserts the asymptotic form of $w_{n}(z)$ eq. (B.5) into the integral on the right hand side of the recurrence relation (3.15) one obtains the asymptotic form of $w_{n+1}(z)$ as given in (B.5).

\section{Asymptotic of the hypergeometric kernel}

In this appendix we discuss the asymptotic behaviour of the truncated sum in the kernel eq. (4.3). The asymptotic of the corresponding infinite sum was discussed in reference [28, and we again give some details here to be self-contained. Let us repeat the definition

$$
T_{n}(x, N)=\sum_{k=0}^{N-1} \frac{x^{k}}{(\pi k !)^{n}},
$$

which we would like to investigate for large $N$ and for $x$ of order $N^{n}$, due to $x=|z|^{2} \lesssim N^{n}$. We again apply the saddle point method. By first using Stirling's formula $k ! \approx \sqrt{2 \pi k}(k / e)^{k}$ and then approximating the sum by an integral we obtain:

$$
T_{n}(x, N) \approx\left(2 \pi^{3}\right)^{-n / 2} \int_{1}^{N} d k k^{-n / 2} \mathrm{e}^{s(k)}
$$

where $s(k)=k \ln [x]-n k \ln [k]+n k$. We are interested in the behaviour of $T_{n}(x, N)$ for large $N$ and $x$. In this case the exponent $\mathrm{e}^{s(k)}$ can be approximated by a Gaussian function with a maximum located at

$$
k_{*}=x^{1 / n}
$$

being a solution of the saddle point equation

$$
s^{\prime}\left(k_{*}\right)=\ln [x]-n \ln \left[k_{*}\right]=0 .
$$


We also have $s\left(k_{*}\right)=n k_{*}, s^{\prime \prime}\left(k_{*}\right)=-n / k_{*}$, so the Gaussian approximation gives:

$$
T_{n}(x, N) \approx\left(2 \pi^{3}\right)^{-n / 2} \mathrm{e}^{n k_{*}} \int_{1}^{N} d k k^{-n / 2} \mathrm{e}^{-n\left(k-k_{*}\right)^{2} /\left(2 k_{*}\right)},
$$

with $k_{*}=x^{1 / n}$ (C.3). Let us change the integration variable in the last integral to $t=\sqrt{n / 2 k_{*}}\left(k-k_{*}\right)$. Written in this new variable the Gaussian part assumes the form $\mathrm{e}^{-t^{2}}$, which means that values of $t$ that contribute to the integral are of order one. In this narrow range of $t$, the factor $k^{-n / 2}$ can be treated as constant. Indeed, for $t$ of order of unity and $k_{*}$ of order $N$, we have $k=k_{*}\left(1+t /\left(n k_{*} / 2\right)^{1 / 2} \approx k_{*}\right.$, for $t \ll\left(n k_{*} / 2\right)^{1 / 2}$. The upper integration limit for $t$ is: $\sqrt{n / 2 k_{*}}\left(N-k_{*}\right)$. The lower one is far below the range of the Gaussian integrand, so it can be set to $-\infty$. Replacing $k_{*}$ by $x^{1 / n}$ (C.3) we eventually obtain

$$
\lim _{N>k_{*} \gg 1} T_{n}(x, N) \approx \frac{1}{\pi \sqrt{n}}\left(2 \pi^{3}\right)^{(1-n) / 2} x^{(1-n) / 2 n} \exp \left[n x^{1 / n}\right] \frac{1}{2} \operatorname{erfc}\left(\frac{\sqrt{n}\left(x^{1 / n}-N\right)}{\sqrt{2 x^{1 / n}}}\right) .
$$

In the limit $N \rightarrow \infty$ the truncated sum becomes an infinite series $T_{n}(x)=\lim _{N \rightarrow \infty} T_{n}(x, N)$. In this case the saddle point is always located within the range of integration, so we have

$$
T_{n}(x)=\sum_{k=0}^{\infty} \frac{x^{k}}{(\pi k !)^{n}} \stackrel{x \gg 1}{\approx} \frac{1}{\pi \sqrt{n}}\left(2 \pi^{3}\right)^{(1-n) / 2} x^{(1-n) / 2 n} \exp \left[n x^{1 / n}\right] .
$$

\section{Eigenvalue distribution of $\mathbf{n}$ powers of a single Ginibre matrix}

In this appendix we discuss the eigenvalue distribution of the $n$-th power of a single Ginibre matrix. Consider a random matrix $A$ with a spherically symmetric eigenvalue distribution $\rho(w)=\rho(|w|=r)$. Denote by $\tilde{\rho}_{n}(w)$ the corresponding spectral density of $A^{n}$ - the $n$-th power $A$, which is also angleindependent. If $\lambda$ is an eigenvalue of $A$, then $\tilde{\lambda}=\lambda^{n}$ is an eigenvalue of $A^{n}$. Changing the integration variable in the formula below to $s=r^{n}$, we have:

$$
\int_{0}^{\infty} 2 \pi r \rho(r) d r=\int_{0}^{\infty} \frac{2 \pi s^{2 / n-1}}{n} \rho\left(s^{1 / n}\right) d s=\int_{0}^{\infty} 2 \pi s \tilde{\rho}_{n}(s) d s,
$$

and thus

$$
\tilde{\rho}_{n}(r)=\frac{r^{\frac{2}{n}-2}}{n} \rho\left(r^{1 / n}\right)
$$

In particular, for the limiting macroscopic density of a single Ginibre matrix $X$ with $n=1$ we have $\rho_{\text {macro }}^{(1)}(w)=\frac{1}{\pi} \Theta(1-|w|)$ and therefore the corresponding density of its $n$-power is

$$
\tilde{\rho}_{\text {macro }}(w)=\frac{|w|^{\frac{2}{n}-2}}{\pi n} \Theta(1-|w|) .
$$

This is exactly as for the product of $n$-independent matrices (5.5). We can also repeat calculations for the finite size distribution for a single Ginibre matrix in eq. (5.3) :

$$
\rho(w)=N^{-1} R_{1}^{(1)}\left(N^{\frac{1}{2}} w\right)=\frac{1}{2 \pi} \operatorname{erfc}(\sqrt{2 N}(|w|-1)) .
$$

Applying (D.2) we find the corresponding distribution of eigenvalues of its $n$-th power:

$$
\tilde{\rho}_{n}(w)=\frac{|w|^{\frac{2}{n}-2}}{\pi n} \frac{1}{2} \operatorname{erfc}\left(\sqrt{2 N}\left(|w|^{1 / n}-1\right)\right) .
$$


It can be further simplified using a Taylor expansion at $|w|=r=1$ to approximate the function inside the erfc-function: $r^{1 / n}-1=(1 / n)(r-1)+\ldots$ and neglecting higher order terms, as in section 5 ;

$$
\tilde{\rho}_{n}(w)=\frac{|w|^{\frac{2}{n}}-2}{\pi n} \frac{1}{2} \operatorname{erfc}\left(\frac{\sqrt{2 N}}{n}(|w|-1)\right) .
$$

An important difference between this case and the power of $n$ independent matrices is that the dependence on $n$ in the denominator is $n$, and not $\sqrt{n}$ as in eq. (5.3).

\section{References}

[1] G. Akemann, J. Baik, P. Di Francesco (Eds.), "The Oxford Handbook of Random Matrix Theory", Oxford University Press, Oxford 2011.

[2] J. Ginibre, J. Math. Phys. 6 (1965) 440-449.

[3] H. Furstenberg and H. Kesten, Ann. Math. Stat. 31 (1960) 457-469.

[4] K. A. Penson, K. Życzkowski, Phys. Rev. E83 (2011) 061118 [arXiv:1103.3453v3 [math-ph]].

[5] P. J. Forrester, Lyapunov exponents for products of complex Gaussian random matrices, arXiv:1206.2001 v1 [math.PR].

[6] J. C. Osborn, Phys. Rev. Lett. 93 (2004) 222001 hep-th/0403131.

[7] Z. Burda, R. A. Janik, B. Waclaw, Phys. Rev. E81 (2010) 041132 arXiv:0912.3422v2 [condmat.stat-mech]].

[8] F. Götze, A. Tikhomirov, On the Asymptotic Spectrum of Products of Independent Random Matrices, arXiv:1012.2710v3 [math.PR].

[9] Z. Burda, A. Jarosz, G. Livan, M. A. Nowak and A. Swiech, Phys. Rev. E82 (2010) 061114 arXiv:1007.3594v1 [cond-mat.stat-mech]]; Acta Phys. Polon. B42 (2011) 939-985 arXiv:1103.3964v1 [cond-mat.stat-mech]].

[10] S. O'Rourke, A. Soshnikov, Electr. J. Prob. 81 (2011) 2219-2245 [arXiv:1012.4497v3 [math.PR]].

[11] G. Akemann, Nucl. Phys. B730 (2005) 253-299 [hep-th/0507156].

[12] G. Akemann, M. J. Phillips and H.-J. Sommers, J. Phys. A: Math. Theor. 42 (2009) 012001 arXiv:0810.1458v1 [math-ph]]; J. Phys. A43 (2010) 085211 [arXiv:0911.1276 [hep-th]].

[13] G. Akemann, Acta Phys. Polon. B42 (2011) 901-921 [arXiv:1104.5203v1 [math-ph]].

[14] G. Akemann, M. J. Phillips, L. Shifrin, J. Math. Phys. 50 (2009) 063504 arXiv:0901.0897v2 [math-ph]].

[15] E. Kanzieper and N. Singh, J. Math. Phys. 51 (2010) 103510 [arXiv:1006.3096v2 [math-ph]].

[16] P. J. Forrester and G. Honner, J. Phys. A32 (1999) 2961 cond-mat/9812388.

[17] E. Kanzieper, J. Phys. A: Math. Gen. 35 (2002) 6631 cond-mat/0109287. 
[18] B. A. Khoruzhenko and H.-J. Sommers, "Non-Hermitian Random Matrix Ensembles", chapter 18 in [1] arXiv:0911.5645[math-ph]].

[19] E. Bogomolny, J. Phys. A: Math. Theor. 43 (2010) 335102 arXiv:0912.5444v1 [math-ph]].

[20] J. Fischmann, W. Bruzda, B. A. Khoruzhenko, H.-J. Sommers, K. Życzkowski, J. Phys. A45 (2012) 075203 [arXiv:1107.5019v2 [math-ph]].

[21] Y. Ameur, H. Hedenmalm, and N. Makarov, Duke Math. J. 159 No 1 (2011) 31-81 arXiv:0807.0375v3 [math.PR]].

[22] T. Tao, V. Vu, Random matrices: Universality of local spectral statistics of non-Hermitian matrices, arXiv:1206.1893v2 [math.PR].

[23] M. L. Mehta, "Random Matrices", Academic Press, Third Edition, London 2004.

[24] G. H. Golub, C. F. Van Loan, "Matrix Computations", The John Hopkins University Press, Second Edition, London 1993.

[25] I. S. Gradshteyn and I. M. Ryzhik, "Table of Integrals, Series, and Products", Academic Press, San Diego 2000.

[26] J. R. Klauder, K.A. Penson, J.-M Sixdeniers, Phys. Rev. A 64 (2001) 013817.

[27] J. L. Fields, Math. Comp. 119 vol. 29 (1972) 757-765.

[28] Z. Burda, J. Jurkiewicz, A. Krzywicki, Phys. Rev. E69 (2004) 026106 arXiv:cond-mat/0310234.

[29] Z. Burda, M.A. Nowak, A. Swiech, New spectral relations between products and powers of isotropic random matrices, arXiv:1205.1625v2 [cond-mat.stat-mech]. 